\title{
comportement à terme des terrains boulonnés par scellements répartis à la résine
}

\section{J.-F. Raffoux}

Docteur-Ingénieur

CERCHAR

Groupe Terrains

Verneuil-en-Halatte

Laboratoire de Mécanique des Terrains

Nancy 


\section{COMPORTEMENT A TERME DES TERRAINS BOULONNES PAR SCELLEMENTS REPARTIS A LA RESINE}

L'utilisation de scellements répartis par résines synthétiques pour le boulonnage des terrains est une technique récente. Son développement a eu lieu tout d'abord dans les travaux souterrains miniers dont la durée de vie est en général assez courte. Son utilisation dans les travaux souterrains de génie civil à longue durée de vie pose le problème de l'efficacité à terme de ce type de renforcement.

Des observations et mesures faites dans des galeries de mines restées ouvertes depuis plusieurs années ( 3 à 6 ans) montrent que sur cette durée les terrains boulonnés houillers restent stables et que les scellements y conservent toute leur efficacité.
MIDDLE TERM BEHAVIOUR OF STRATA REINFORCED BY FULL COLUMN RESIN BOLTS

Strata reinforcement by full column bolting is still a new technic. It was first developped in mine workings caracterised by a rather short life span. Its extention to tunelling and civil engeneering can only be undertaken after long term efficiency has been studied.

Measurements and observations in some mine workings which have been open for several years ( 3 to 6$)$ show that after that time the strata reinforced with resin bolts remain stable, and bolts are still efficient.

Comité Français de Mécanique des Roches : exposé du 19 juin 1975. 


\title{
comportement à terme des terrains boulonnés par scellements répartis à la résine
}

\author{
par J.-F. RAFFOUX
}

L'utilisation de scellements répartis par résines polyesters pour le boulonnage des terrains est une technique récente puisque son application industrielle date d'une dizaine d'années.

Son développement a essentiellement eu lieu dans les travaux souterrains miniers qui en ont consommé la presque totalité jusqu'à ces tous derniers temps. Les travaux publics souterrains commencent à utiliser cette technique mais l'emploi de ces scellements dans des ouvrages permanents pose le problème de leur longévité, problème qui ne se posait pas dans les voies de mines qui ont généralement des durées de vie assez courtes (de l'ordre d'un an).

Les chimistes estiment que la résine polyester polymérisée est un produit stable, mais on ne dispose encore d'aucune information expérimentale sur le comportement à terme dans le terrain de ces scellements.

Or, dans les Charbonnages de France, plusieurs exploitations ont avec l'aide du CERCHAR mis au point ces techniques de scellements répartis depuis plus de dix ans. En particulier, les Houillères du bassin de Lorraine font une utilisation extensive du « boulonnage à la résine "; près de $6 \mathrm{~km}$ de voies et de galeries sont creusées chaque année en utilisant cette technique éventuelle complétée par un soutènement porteur additionnel indépendant et certaines restent ouvertes et accessibles pendant plusieurs années. Dans six de ces voies et galeries creusées depuis longtemps divers essais et mesures ont permis de vérifier l'efficacité à terme du boulonnage par scellements polyesters.

\section{PRESENTATION}

DES

Les six ouvrages qui ont permis d'étudier le comportement à terme de scellements à la résine et des terrains boulonnés ont été sélectionnés d'après les critères suivants :

- Ils étaient creusés et boulonnés depuis plus de trois ans.

- Ils comportaient des dispositifs de mesures d'expansion destinés au contrôle systématique des défor-

\section{OUVRAGES ETUDIES}

mation au creusement et qui soient encore accessibles.

- Ils offraient la possibilité d'y effectuer des essais de traction sur quelques boulons de façon à tester les performances du scellement après quelques années.

Trois voies au charbon, deux galeries et une carrure au rocher répondaient à ces critères. Leurs caractédistiques sont rassemblées dans le tableau ci-après :

\begin{tabular}{|c|c|c|c|c|c|}
\hline $\begin{array}{l}\text { Localisation } \\
\text { profondeur }\end{array}$ & Age & $\begin{array}{l}\text { Type d'ouvrage } \\
\text { Nature des terrains }\end{array}$ & $\begin{array}{l}\text { Influence subie } \\
\text { après creusement }\end{array}$ & $\begin{array}{l}\text { Nombre d'essais } \\
\text { de traction } \\
\text { réalisés }\end{array}$ & $\begin{array}{c}\text { Nombre de stations } \\
\text { de mesures } \\
\text { de déformations } \\
\text { en état }\end{array}$ \\
\hline $\begin{array}{l}\text { Folschwiller } \\
\text { Maurice } 18-1 \\
\qquad 870 \mathrm{~m}\end{array}$ & 5 ans & $\begin{array}{l}\text { Voie en veine } \\
\text { Toit : banc épais grès } \\
\text { Mur : tendre, bancs } \\
\text { minces de charbon } \\
\text { et schiste }\end{array}$ & $\begin{array}{l}\text { Démarrage de tail. } \\
\text { parallèlement à la voie } \\
\text { à } 30 \mathrm{~m}\end{array}$ & 5 & 1 \\
\hline $\begin{array}{l}\text { Folschwiller } \\
\text { Carrures } \\
\qquad 870 \mathrm{~m}\end{array}$ & 6 ans & $\begin{array}{l}\text { Galeries et carrures } \\
\text { d'infrastructure } \\
\text { au rocher - schistes }\end{array}$ & $\begin{array}{l}\text { Passage d'exploitation } \\
\text { latéralement à } 100 \mathrm{~m}\end{array}$ & 3 & 4 \\
\hline $\begin{array}{l}\text { Folschwiller } \\
\text { Maurice } 22 \\
\qquad 870 \mathrm{~m}\end{array}$ & 6 ans & $\begin{array}{l}\text { Voie en veine } \\
\text { Toit : banc épais grès } \\
\text { Mur : tendre, charbon } \\
\text { et schiste }\end{array}$ & Aucune & 14 & 3 \\
\hline $\begin{array}{l}\text { Folschwiller } \\
\text { Travers bancs } \\
\text { vers Maurice } 22 \\
\quad 870 \mathrm{~m}\end{array}$ & 4 ans & $\begin{array}{l}\text { Galerie au rocher } \\
\text { Schistes gréseux }\end{array}$ & $\begin{array}{l}\text { Passage d'exploitation } \\
\text { latéralement } \\
\text { de } 20 \text { à } 100 \mathrm{~m}\end{array}$ & 3 & 1 \\
\hline $\begin{array}{l}\text { Marienau } \\
\qquad \mathrm{G}_{2} \mathrm{~A} 660 \mathrm{~m}\end{array}$ & 3 ans & $\begin{array}{l}\text { Voie en veine } \\
\text { Toit : grès } \\
\text { Mur : schiste + grès }\end{array}$ & $\begin{array}{l}\text { Passage de taille } \\
\text { remblayée } \\
\text { pneumatiquement } \\
\text { le long de la voie }\end{array}$ & 8 & 1 \\
\hline $\begin{array}{l}\text { Marienau } \\
\qquad \mathrm{G}_{2} \mathrm{~B} 660 \mathrm{~m}\end{array}$ & 3 ans & $\begin{array}{l}\text { Voie en veine } \\
\text { Toit : stratifié } \\
\text { schiste, charbon, grès } \\
\text { Mur : grès }\end{array}$ & Aucune & 17 & 1 \\
\hline
\end{tabular}


Le boulonnage mis en œuvre dans ces galeries présentait les caractéristiques suivantes :

- scellement des boulons sur toute leur longueur $(2.20 \mathrm{~m})$;

- boulons posés dès la mise à nu des terrains (une à cinq heures après le tir) ;

- charges de scellement constituées de résine polyester thermodurcissable $(30 \%)$ et d'une charge neutre (dolomie). Accélérateur + catalyseur (peroxyde de benzoïle);
- tiges d'acier nervuré AC 60 de diamètre $20 \mathrm{~mm}$ dans trou de diamètre $28 \mathrm{~mm}$;

- foration au marteau perforateur (semi stopper). Introduction de la tige du boulon à la perforatrice ;

- garnissage continu du toit et des parements par grillage ;

- serrage sur plaque (15 à $20 \mathrm{~m} \mathrm{~kg})$.

La figure 1 représente les schémas de trois des voies et galerie retenues. Ce sont des schémas couramment adoptés par les Houillères du Bassin de Lorraine.
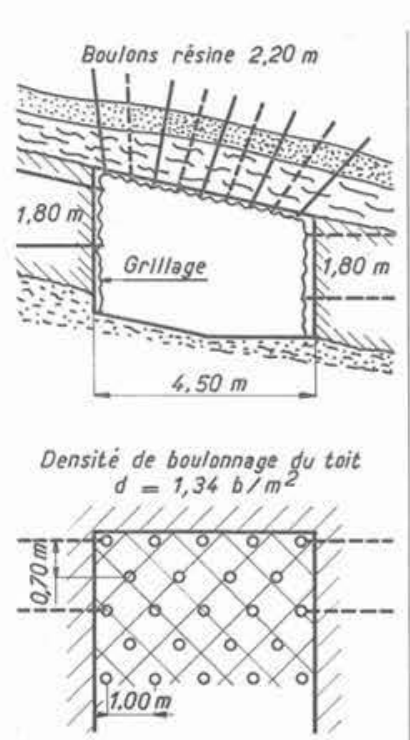

siege de Folschviller Voie de base - Maurice 22

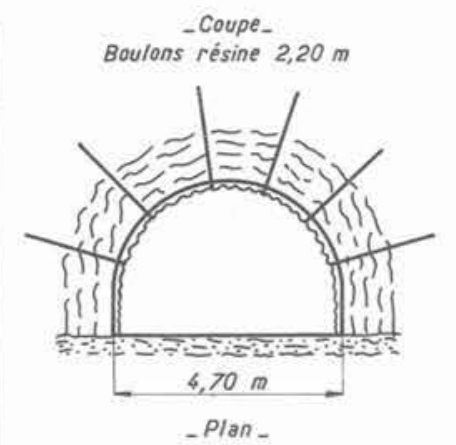

Densité de boulonnage du toit $d=0,66 \mathrm{~b} / \mathrm{m}^{2}$

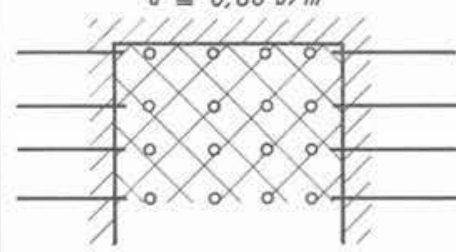

siège de Folschviller

Travers banc vers Maurice 22

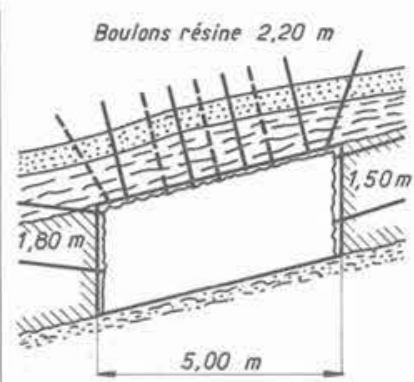

Densité de boulonnage du toit

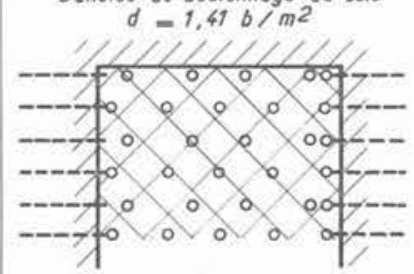

Siège de Folschviller

Voie de base - Maurice 22

Fig. 1. - Schémas de boulonnage types de voies et galeries aux Houillères du Bassin de Lorraine.

\section{COMPORTEMENT DES TERRAINS AUTOUR DES OUVRAGES ETUDIES}

Comme toutes les voies boulonnées des Houillères du Bassin de Lorraine, les six voies et galeries retenues avaient été équipées dès le creusement de stations de mesures de l'expansion du toit au milieu de la voie (fig, 2).

La pose de ces stations a pour objectif un contrôle des déformations qui consiste à comparer leurs valeurs à des standards d'amplitude ou d'accélération. En cas de dépassement de ces standards des mesures de renforcement sont prises pour éviter l'évolution vers un processus d'éboulement. Le traitement quotidien de ces mesures est réalisé avec l'aide d'un ordinateur.

Il y avait dans chacun des six ouvrages de dix à vingt stations de contrôle posées au creusement; les

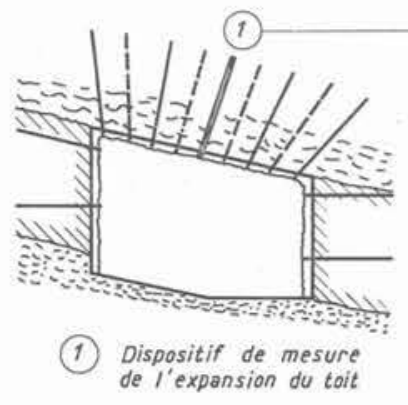

Fig. 2. - Station de contrôle de l'expansion du toit.

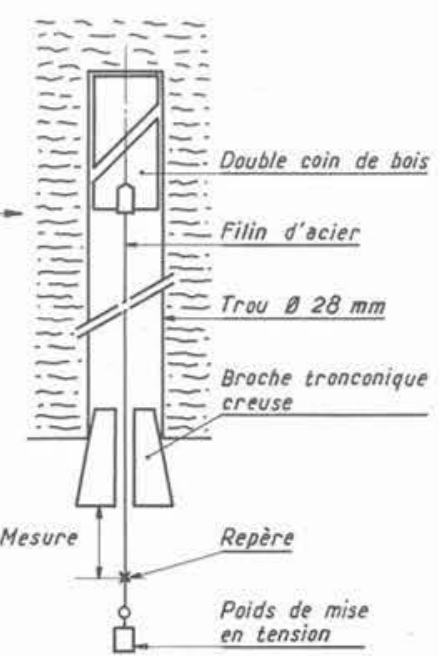


dispositifs de mesures équipant ces stations de contrôle sont simples (fig. 2 b), peu coûteux mais, réalisés avec des matériaux altérables (bois, fil d'acier), ils ne sont pas prévus pour avoir une longue durée de vie. Cependant, un certain nombre de stations en état ont été retrouvées dans cinq des six ouvrages étudiés. Trois seulement dans les plus anciennes des voies (six ans) mais 14 et 17 dans les plus récentes (trois ans). Sur les dispositifs en bon état, qui sont presque toujours des dispositifs d'expansion de nouvelles mesures ont été entreprises; confrontées aux mesures prises plusieurs années auparavant, elles permettent de comparer l'expansion qui s'est produite au moment du creusement à celle qui s'est produite par la suite (Tableau 1).

Avant l'analyse de ce tableau, il faut déjà noter que, la faible amplitude des déformations observées dans plusieurs stations de mesure (quelques millimètres) est de l'ordre de grandeur de la précision de la mesure.

L'examen d'ensemble de ces résultats de mesures des déformations montre donc :

- que les voies Maurice 18, Maurice 22 et le travers banc vers Maurice 22 ont depuis la fin du creusement des expansions très faibles voire nulles. Lors du creusement les expansions étaient déjà faibles (inférieures à $10 \mathrm{~mm}$ );

- que la voie $G_{2} A$ où les expansions étaient faibles au creusement (inférieures à $10 \mathrm{~mm}$ ) et fortes au passage taille (en moyenne $50 \mathrm{~mm}$ ) a, sauf pour une station, des expansions faibles par la suite ;

- que la voie $G_{2} B$ où les expansions étaient assez fortes dès le creusement a continué à avoir des expansions assez fortes par la suite.

De cet échantillon limité mais représentatif, il ressort que :

Dans les toits de grès et de schiste gréseux où le boulonnage a eu une efficacité instantanée en bloquant les expansions dès le creusement, il n'y a pratiquement pas d'évolution des terrains sous la seule influence du temps.

Dans le toit de la voie $G_{2} A$ qui, lors du passage taille, a eu des expansions assez fortes mais normales dans cette phase de fortes sollicitations, les expansions ont très peu évoluées. Le boulonnage et le soutènement porteur additionnel mis en place au passage de la taille contrôlent donc de façon satisfaisante l'évolution dans le temps du comportement de ce milieu fissuré et fracturé.

\begin{tabular}{|c|c|c|c|c|c|c|}
\hline \multirow{2}{*}{$\begin{array}{l}\text { Ouvrages et } \\
\text { nombre de sta } \\
\text {-tions de mesu } \\
\text { res en état } \\
\end{array}$} & \multicolumn{2}{|c|}{$\begin{array}{c}\text { Exponsion } \\
\text { ou creusement }\end{array}$} & \multicolumn{2}{|c|}{$\begin{array}{c}\text { Expansion } \\
\text { au passage taille }\end{array}$} & \multicolumn{2}{|c|}{$\begin{array}{l}\text { Expansion depuis } \\
\text { fin de l'exploptation }\end{array}$} \\
\hline & (1) & (2) & (1) & (2) & (1) & (2) \\
\hline & $\begin{array}{l}2 \\
1 \\
2\end{array}$ & $\begin{array}{l}3 \text { mois } \\
3 \text { " } 2 \text { sem. } \\
2 . "\end{array}$ & $\bar{z}$ & $\bar{z}$ & $\begin{array}{l}1 \\
2 \\
6\end{array}$ & $\begin{array}{l}50 \text { mois } \\
66 \% " 2 \text { sem } \\
60 " \text { " }\end{array}$ \\
\hline & $\begin{array}{l}1 \\
1 \\
6 \\
4 \\
6 \\
6\end{array}$ & 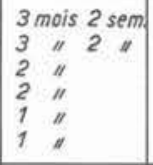 & $\begin{array}{l}\bar{z} \\
\bar{z}\end{array}$ & $\begin{array}{l}\bar{z} \\
\bar{z}\end{array}$ & $\begin{array}{l}0 \\
0 \\
0 \\
0 \\
6 \\
2\end{array}$ & $\begin{array}{lll}32 & \text { mois } & \\
32 & \prime \prime & 2 \text { sem } \\
32 & \prime \prime & " \prime \\
30 & \prime \prime & \\
30 & \prime \prime & \\
28 & \text { "l } & 2 \text { sem }\end{array}$ \\
\hline & $\begin{array}{l}5 \\
0 \\
0\end{array}$ & $\begin{array}{ll}1 & 11 \\
1 & 11 \\
1 & 11\end{array}$ & $\bar{z}$ & $\bar{z}$ & 5 & $\begin{array}{l}33 \text { mois } 2 \text { sem } \\
41 " ~ \\
30 " 1\end{array}$ \\
\hline $\begin{array}{l}\text { Mar } \\
6 \\
11 \text { st }\end{array}$ & $\begin{array}{r}3 \\
8 \\
20 \\
11 \\
15 \\
6 \\
24 \\
15 \\
12 \\
8 \\
2\end{array}$ & 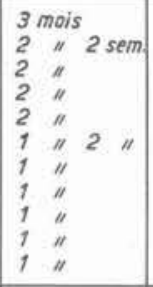 & $\begin{array}{l}\bar{z} \\
\bar{z} \\
\bar{z} \\
\bar{z}\end{array}$ & $\begin{array}{l}\bar{z} \\
\bar{z} \\
= \\
= \\
=\end{array}$ & $\begin{array}{r}0 \\
14 \\
0 \\
37 \\
32 \\
40 \\
2 \\
43 \\
30 \\
70 \\
75\end{array}$ & $\begin{array}{l}35 \text { mois } \\
37 \text { " } \\
32 \text { " } \\
33 \text { " } 1 \text { sem } \\
33 \text { " } 1 \text { " } \\
33 \text { " }\end{array}$ \\
\hline $\begin{array}{c}\text { Marienau } \\
G_{2} A \\
8 \text { stations }\end{array}$ & $\begin{array}{l}1 \\
0 \\
4 \\
4 \\
3 \\
2 \\
4 \\
5\end{array}$ & $\begin{array}{llll}3 & \text { mois } 1 & \text { sem } \\
5 & \prime \prime & 3 & \prime \prime \\
5 & \prime \prime & 3 & \prime \prime \\
6 & \prime \prime & & \\
6 & \prime \prime & 3 & \prime \prime \\
4 & \prime \prime & & \\
5 & \prime \prime & & \end{array}$ & $\begin{array}{r}160 \\
19 \\
52 \\
40 \\
38 \\
18 \\
20 \\
56\end{array}$ & $\begin{array}{llll}6 & \text { mois } & 2 & \text { sem } \\
6 & 11 & 1 & \prime \prime \\
5 & \prime \prime & 3 & \prime \prime \\
6 & \prime \prime & 2 & \prime \prime \\
5 & 11 & 2 & \text { " } \\
5 & \prime \prime & & \\
5 & \prime \prime & & \\
5 & \prime \prime & & \end{array}$ & $\begin{array}{r}0 \\
0 \\
7 \\
0 \\
2 \\
12 \\
5 \\
06\end{array}$ & 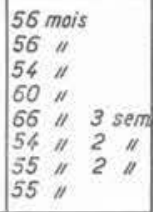 \\
\hline
\end{tabular}

(1) Valeurs absolues $(\mathrm{mm})$ de l'expansion dans chaque phase

(2) Durée de la phase en mois et semaine

TABLEAU 1

Expansion des terrains lors des phases d'exploitation et depuis la fin de celle-ci

Le toit de $G_{2}$ B qui est stratifié avec alternance de schistes, de grès et charbon a eu des expansions normales pour ce type de toit et dès le creusement le boulonnage a été efficace pour en contrôler l'évolution. Cette efficacité a été durable puisque qu'avec l'aide d'un soutènement porteur additionnel très réduit, les expansions bien que non négligeables n'ont pas conduit à la ruine du toit.

\section{ESSAIS DE TRACTION SUR DES BOULONS}

L'essai de traction ne simule absolument pas le comportement du boulon dans le terrain mais c'est un moyen commode de tester l'efficacité d'un scellement ou d'un ancrage. La figure 3 a présente le schéma d'installation d'un tel essai.

Onze essais de traction ont pu être effectués en 1975 sur des boulons qui avaient été posés lors du creusement des ouvrages étudiés; deux ont été réalisés sur des boulons posés dans le charbon des parements et les neuf autres sur des boulons de toit ou de parements de travers banc ou carrures.

Ne disposant pas de résultats d'essais de traction réalisés dans ces mêmes ouvrages lors de la pose des boulons au creusement, il a fallu comparer leur résultats à ceux d'essais de traction sur des boulons récemment posés dans des terrains semblables et avec les mêmes caractéristiques et modes opératoires. Douze résultats d'essais de traction récents ont donc été utilisés pour la comparaison.

Les courbes des essais de traction représentent le déplacement relatif de l'extrêmité du boulon par rapport au vérin (c'est-à-dire l'allongement de la tige, son glissement, et éventuellement l'enfoncement du vérin dans le terrain) en fonction de l'effort qui lui est appliqué ; ces courbes (fig. $3 \mathrm{~b}$ ) présentent habituellement une phase linéaire ou pseudo linéaire qui est parfois précédée d'une phase de mise en serrarge du vérin par écrasement superficiel des terrains. Vers $15 \mathrm{t}$, on constate souvent un changement de pente de la courbe effort-déplacement et l'essai se termine par la rupture de la tige à moins qu'il n'ait été interrompu avant par crainte de dépasser la limite d'utilisation du vérin $(20 \mathrm{t})$. 


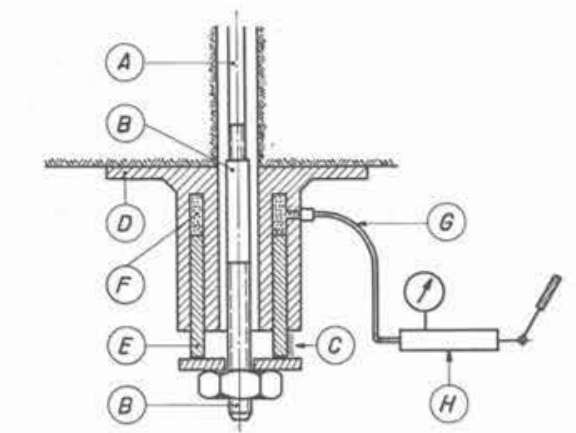

A - Tige du boulon essayé
B - Tube rallonge fileté
C - Lecture de I'allongement (d)
O - Corps du verrin creux
E - Piston
F - Huile
- Flexible de raccordement i la pompe
H - Pompe et manomètre de lecture de
la pression appliquée $(P)$ ou de l'effort $(F)$
Fig.3a-Vérin annulaire de traction sur boulon

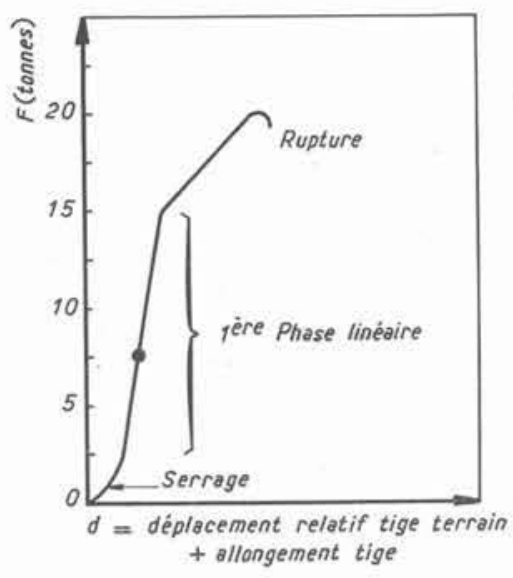

Fig. 3. - Essai de traction.

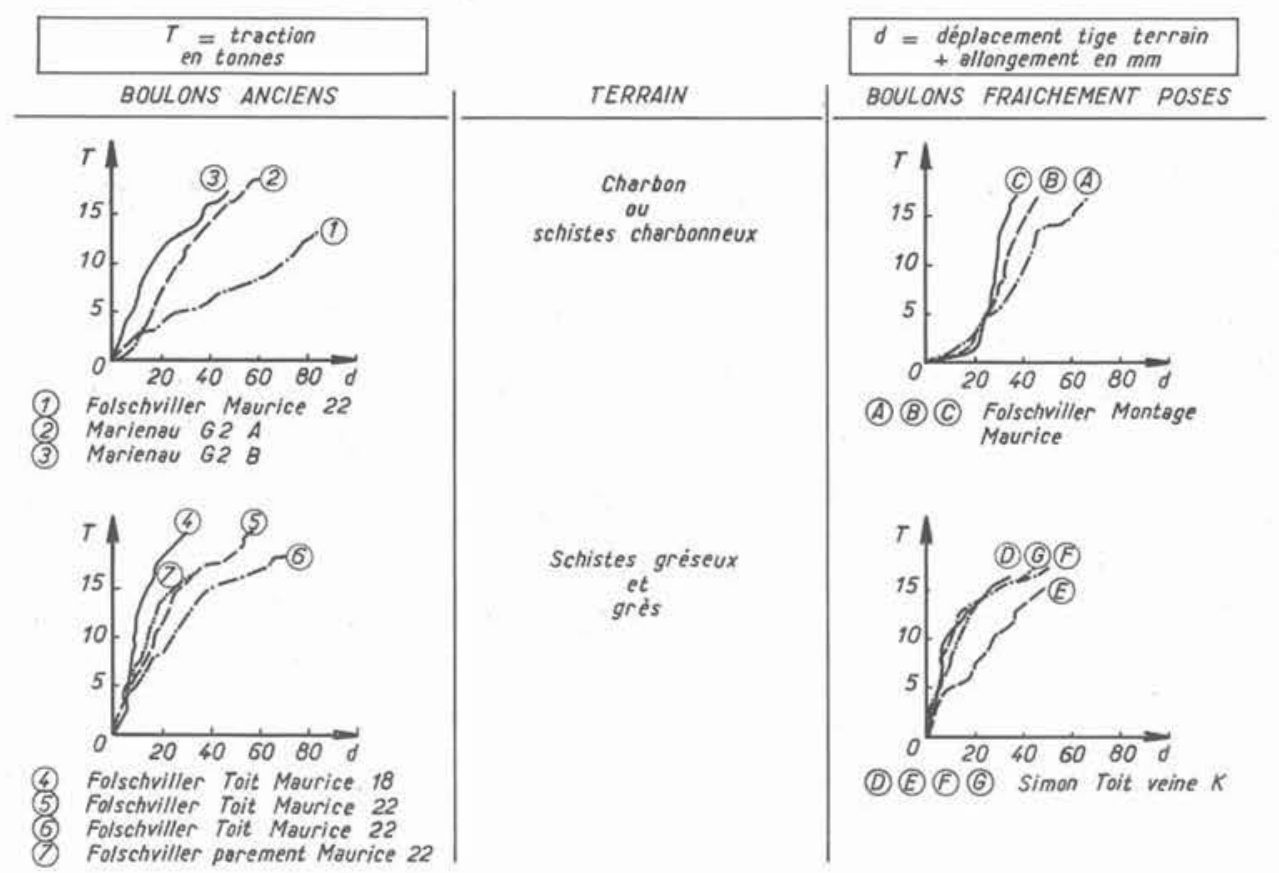

Fig. 4. - Exemples de courbes d'essais de traction.

Les courbes de la figure 4 et le tableau 2 présentent les principaux résultats de ces essais de traction et en particulier la pente de la première phase linéaire de la courbe effort-déplacement qui dans chaque essai caractérise le comportement de l'ensemble tige-résineterrain sous un effort de traction.
Les moyennes de ces pentes sont très voisines pour les deux catégories de boulons : $7 \mathrm{~T} / \mathrm{cm}$ pour les boulons anciens et $7.5 \mathrm{~T} / \mathrm{cm}$ pour les boulons récemment posés et plus généralement l'analyse statistique ne permet pas de mettre en évidence de différence de comportement entre des boulons scellés à la résine depuis plusieurs années et ceux des boulons à scellement récent. 


\begin{tabular}{|c|c|c|c|c|c|c|}
\hline \multicolumn{3}{|c|}{ BOULONS ANCIENS } & \multirow[b]{2}{*}{ Terrain } & \multicolumn{3}{|c|}{ BOULONS FRAICHEMENT POSES } \\
\hline $\begin{array}{c}\text { Age } \\
\text { du } \\
\text { boulon }\end{array}$ & $\begin{array}{l}\text { Pente } \\
\text { de la phase } \\
\text { linéaire } \\
\end{array}$ & $\begin{array}{c}\text { Charge limite } \\
\text { atteinte }\end{array}$ & & $\begin{array}{l}\text { Age } \\
\text { du } \\
\text { boulon }\end{array}$ & $\begin{array}{l}\text { Pente } \\
\text { de la phase } \\
\text { linéaire }\end{array}$ & $\begin{array}{c}\text { Charge limite } \\
\text { atteinte }\end{array}$ \\
\hline $\begin{array}{l}3 \text { ans } \\
4 \text { ans } \\
5 \text { ans }\end{array}$ & $\begin{array}{l}1,5 \mathrm{t} / \mathrm{cm} \\
3 \mathrm{t} / \mathrm{cm} \\
5 \mathrm{t} / \mathrm{cm}\end{array}$ & $\begin{array}{l}13 \mathrm{t} \text { Vérin s'enfonce } \\
18 \mathrm{t} \text { Rupture tige } \\
18 \mathrm{t} \text { Arrêt essai }\end{array}$ & $\begin{array}{c}\text { Charbon } \\
\text { et } \\
\text { schistes charbonneux }\end{array}$ & $\simeq 1$ heure & $\begin{array}{cc}4.5 & t / \mathrm{cm} \\
6 & t / \mathrm{cm} \\
15 & t / \mathrm{cm}\end{array}$ & 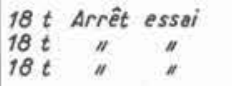 \\
\hline 6 ans & $\begin{array}{l}9 \mathrm{t} / \mathrm{cm} \\
5,5 \mathrm{t} / \mathrm{cm} \\
3,5 \mathrm{t} / \mathrm{cm} \\
20 \mathrm{t} / \mathrm{cm}\end{array}$ & $\begin{array}{l}21,5 t \text { Arrêt essai } \\
9,5 t \text { Rupture tige } \\
21,5 t \text { Arrêt essai } \\
19 \text { t Rupture tige }\end{array}$ & Schistes & & & \\
\hline \multirow[t]{2}{*}{3 ans } & $\begin{array}{rl}10 & t / \mathrm{cm} \\
6 & t / \mathrm{cm} \\
7 & t / \mathrm{cm}\end{array}$ & $\begin{array}{llll}10 & t & \text { Arrêt essai } \\
21 & t & \text { Arrêt } & \text { essai } \\
17 & t & \text { Arrêt } & \text { essai }\end{array}$ & $\begin{array}{c}\text { Schistes gréseux } \\
\text { et } \\
\text { grès }\end{array}$ & 8 jours & $\begin{array}{cc}7 & t / \mathrm{cm} \\
8 & t / \mathrm{cm} \\
12,5 & t / c m \\
4,5 & t / c m \\
5 & t / c m \\
9 & t / c m \\
2,5 & t / c m \\
4 & t / c m \\
12 & t / c m\end{array}$ & \multirow{2}{*}{$\begin{array}{lll}17 & t & \text { Arrêt essai } \\
17 & t & \text { Arrêt essai } \\
17 & t & \text { Arrêt essai } \\
12 & t & \text { Boulon glisse } \\
17 & t & \text { Arrêt essai } \\
17 & t & \text { Arrêt essai } \\
14 & t & \text { Vérin s'enfonce } \\
15 & t & \text { Boulon glisse } \\
16 & t & \text { Vérin s'enfonce }\end{array}$} \\
\hline & $\begin{array}{l}\text { Pente moyenne } \\
=7,05 \mathrm{t} / \mathrm{cm} \\
\text { Ecart type } \\
=4,97 \mathrm{t} / \mathrm{cm}\end{array}$ & & & & $\begin{array}{l}\text { Pente moyenne } \\
=7,50 \mathrm{t} / \mathrm{cm} \\
\text { Ecart type } \\
=3,76 \mathrm{t} / \mathrm{cm}\end{array}$ & \\
\hline
\end{tabular}

TABLEAU 2

Résultats des essais de traction sur des boulons scellés à la résine.

\section{OBSERVATIONS SUR L'ETAT DES VOIES ET GALERIES}

Ces ouvrages restés ouverts depuis leur creusement ont fait depuis lors l'objet d'une surveillance continue accompagnée parfois de travaux d'entretien. De plus, une inspection plus systématique et complète de leur état a été entreprise lors des dernières mesures effectuées (essais au vérin, mesures d'expansion). On a pu ainsi restituer l'histoire de la voie et caractériser son état actuel :

\section{Folschwiller voie de base de Maurice 18.1 à 870}

L'état général de la voie est très bon malgré un fluage des parements notable. De plus, un rabassenage de $1 \mathrm{~m}$ a été effectué de façon à garder une hauteur utile suffisante à la voie. Un soutènement médian systématique (bille refendue sur étai de bois médian) a été installé après creusement.

\section{Carrures}

Creusées en grandes dimensions $(6 \mathrm{~m}$ de large et $7 \mathrm{~m}$ de hauteur) et équipées de boulons longs $(3 \mathrm{~m}$ ) en couronne, elles ont été renforcées par pose de boulons supplémentaires et confection d'un pilier bétonné à l'intersection de deux galeries, avant le passage de tailles susjacentes. Leur aspect est resté très bon malgré les sollicitations dues à ces passages d'exploitations voisines.

\section{Voie de base Maurice 22}

Cette voie ouverte depuis quatre ans est dans un excellent état. Le soufflage du mur a cependant rendu nécessaire un rabassenage de $1 \mathrm{~m}$ et quelques étais médians ont été posés de place en place.

\section{Marienau Voie de base $\mathbf{G}_{2} \mathrm{~A}$}

Cette voie creusée en 1970 a subi un passage de taille en 1971. A ce moment, elle a été renforcée par des cadres de bois complets avec un étai médian. L'état du toit est excellent. Malgré la présence d'eau le soufflage n'était pas général dans cette voie où le rabassenage n'a pas été systématique.

\section{Montage de déblocage $\mathbf{G}_{2} \mathbf{B}$}

C'est la seule des voies étudiés qui ait dû recevoir un renforcement systématique plus de deux ans après son creusement. Le soutènement est constitué d'étais médians sur bille refendue tous les mètres. Malgré des expansions non négligeables en certains points, le toit reste bon mais le mur souffle beaucoup.

De l'ensemble de ces observations sur l'état des chantiers après plusieurs années, il ressort que dans tous les cas et moyennant par endroits quelques travaux d'entretien ou de légers renforcements, le boulonnage à la résine a permis de conserver les ouvrages ouverts et en état d'assurer leurs fonctions. La longue expérience que peuvent avoir les mineurs des soutènements classiques par cintres métalliques incite à penser que dans les mêmes conditions, les cintres n'auraient pas permis de conserver ces ouvrages dans un état comparable et en particulier qu'une voie comme $G_{2} B$ se serait certainement refermée sous l'effet du délitage progressif des terrains.

\section{CONCLUSIONS}

L'ensemble de ces résultats de mesures et observations permet dans le cadre de la méthode mise en œuvre de conclure à l'efficacité à terme du boulonnage à la résine. Dans des ouvrages miniers creusés depuis trois à six ans, on a pu en effet montrer :
- que des terrains "boulonnés à la résine " où les déformations sont faibles dès le creusement conservent par la suite un excellent comportement ;

- que des terrains boulonnés à la résine et qui ont subi un important remaniement dû au passage d'une 
taille, gardent par la suite un comportement très satisfaisant ;

- que les boulons à la résine ne peuvent pas complètement s'opposer au délitage des terrains stratifiés et fragiles, l'efficacité du boulonnage y est cependant très nette dès le creusement et se poursuit à plus long terme mais pour des raisons de sécurité on $y$ ajoute dans ce cas un léger soutènement porteur de renforcement.

Ces résultats sur le comportement à terme des terrains boulonnés grâce à des scellements répartis sont positifs. Ils confirment l'expérience qu'ont plusieurs mines qui utilisent cette technique de soutènement et de renfor- cement des terrains; ils complètent quelques résultats expérimentaux ponctuels obtenus dans les mines de fer en suivant l'évolution d'un toit boulonné selon plusieurs techniques et qui concluaient à l'efficacité supérieure à terme du scellement réparti.

Il convient cependant de limiter la portée de ces conclusions au domaine expérimental relativement limité (six ouvrages) à la durée de vie de ces voies et galeries (trois à six ans ce qui est encore du court terme pour un ouvrage de génie civil), à la technique utilisée (matériel, modes opératoires, choix des paramètres du boulonnage) dont l'influence est de premier ordre sur l'efficacité immédiate et à terme des scellements répartis.

\section{REMERCIEMENTS}

Nous remercions les Houillères du bassin de Lorraine et plus particulièrement les ingénieurs et techniciens du service GEOP qui nous ont aidés à recueillir l'information présentée dans cette communication. 